\title{
Determinants of Quality of Life in the Acute Stage Following Stroke
}

\author{
Bo-Ok Jeong ${ }^{1}$, Hee-Ju Kang ${ }^{1}$, Kyung-Yeol Bae', Sung-Wan Kim¹, Jae-Min Kim ${ }^{\circledR}$, \\ II-Seon Shin', Joon-Tae Kim², Man-Seok Park², Ki-Hyun Cho and Jin-Sang Yoon ${ }^{1}$ \\ ${ }^{1}$ Departments of Psychiatry and ${ }^{2}$ Neurology, Chonnam National University Medical School, Gwangju, Korea
}

Objective This study aimed to investigate the factors influence the quality of life (QOL) of survivors of an acute stroke.

Methods For 422 stroke patients, assessments were made within two weeks of the index event. QOL was measured using the World Health Organization Quality of Life-Abbreviated form (WHOQOL-BREF), which has four domains related to physical factors, psychological factors, social relationships, and environmental context. Associations of each four WHOQOL-BREF domain score with socio-demographic characteristics (age, sex, education, marital status, religion, and occupation), stroke severity (National Institutes of Health Stroke Scale), physical disability (Barthel Index), cognitive function (Mini-Mental Status Examination: MMSE), grip strength, and psychological distress (Hospital Anxiety and Depression Scale depression and anxiety subscale: HADS-D and HADS-A) were investigated using the linear regression models.

Results Higher physical domain scores were independently associated with higher MMSE scores, stronger hand-grip strength, and lower HADS-D and HADS-A scores; higher psychological domain scores were independently associated with higher educational level, higher MMSE scores, and lower HADS-D and HADS-A scores; higher social relationships domain scores were independently associated with lower HADS-D and HADS-A scores; and higher environmental domain scores were independently associated with higher educational level, higher MMSE scores, and lower HADS-D scores.

Conclusion Psychological distress and impaired cognitive function were independently associated with lower QOL in patients with acute stroke. However, stroke severity, physical disability and other socio-demographic factors were less significantly associated with QOL. These findings underscore the importance of psychological interventions for improving QOL during the acute phase following stroke.

Psychiatry Investig 2012;9:127-133

Key Words Stroke, Depression, Anxiety, Cognitive function, Quality of life.

\section{INTRODUCTION}

Stroke, a common neurological disorder, is one of the leading causes of mortality. ${ }^{1}$ Additionally, strokes can cause major disabilities and poor quality of life (QOL) in survivors. ${ }^{2}$ QOL has been defined as an individuals' perceptions of their position in life in the context of culture, value system, goals, expectations, standards, and concerns. ${ }^{3}$ It has also been defined in terms of well-being, having purpose in life, autonomy, assumption of meaningful roles, and participation in significant relationships. ${ }^{4}$ Approaches to understanding QOL in patients should encom-

Received: October 29, 2011 Revised: December 20, 2011 Accepted: December 21, 2011 Available online: April 2, 2012

$\triangle$ Correspondence: Jae-Min Kim, MD, PhD

Department of Psychiatry, Chonnam National University Medical School, 5 Hak-dong, Dong-gu, Gwangju 501-746, Korea

Tel: +82-62-220-6143, Fax: +82-62-225-2531

E-mail:jmkim@chonnam.ac.kr

(c) This is an Open Access article distributed under the terms of the Creative Commons Attribution Non-Commercial License (http://creativecommons.org/licenses/by$\mathrm{nc} / 3.0$ ) which permits unrestricted non-commercial use, distribution, and reproduction in any medium, provided the original work is properly cited. pass subjective aspects such as feelings and perceptions as well as physical functioning.

Although stroke has a significant impact on the physical, social, and psychological functioning of individuals, ${ }^{5,6}$ research in this area has focused primarily on physical functioning, and psychosocial factors have been relatively neglected. ${ }^{7,8}$ After survival, many factors may affect QOL in those who have experienced a stroke. In addition to neurological issues such the location of the stroke and the nature of the motor impairment, psychological factors are also important determining QOL in stroke survivors. $^{9-14}$

It has been established that several factors affect QOL in stroke survivors and that QOL emerges from a complex interplay among stroke severity, social support, and health-promoting behaviors. Thus, QOL should be assessed multi-dimensionally; physical (motor deficits, spasticity, ataxia, dysarthria, dysphagia, pain, sleep disturbances, and fatigue), functional (mobility, care required), mental (mood, cognition, satisfaction, and self-perception), and social (work, social network, and so- 
cial role) factors must be considered. ${ }^{15}$ However, the predictors of QOL have been found to differ according to the stage of the stroke. During the 3-12 months after a stroke, age, sex, the presence of comorbid conditions, level of education, type of stroke experienced, concordance (paretic arm), persistent pain, tube feeding, lack of physical exercise, functional status, and depression have been established as predictors of QOL. ${ }^{16-18}$ During the long-term follow-up period after stroke (1-6 years), physical, social, functional, and psychological factors such as age, socioeconomic status, diabetes, angina, unemployment in young adults, employment as a manual worker, physical functioning, urinary incontinence, fatigue, stroke severity, depression, and cognition have been identified as contributors to the QOL of stroke survivors. ${ }^{19-22}$ Unfortunately, few studies have identified the factors associated with QOL in stroke survivors during the acute phase after a stroke. Investigating the factors associated with QOL in patients with acute stroke might improve the quality of care of acute stroke.

This study aimed to investigate determinants of QOL during the acute phase following a stroke in a cohort of Korean patients. We evaluated the association of QOL with socio-demographic characteristics and clinical factors such as stroke severity, physical disability, cognitive functions, grip strength, and psychological distress.

\section{METHODS}

\section{Study overview}

This analysis was conducted as a component of a larger parent study investigating mental disorders in stroke survivors using a naturalistic prospective design. The detailed design has been published. ${ }^{23}$ In brief, participants were consecutively recruited from all patients who had recently suffered an ischemic stroke and who were hospitalized in the Department of Neurology of Chonnam National University Hospital (CNUH) in Gwangju, South Korea. Assessments were conducted at 2 weeks and at 1 year after the stroke to investigate the acute and chronic consequences of this event. Recruitment for the initial 2week assessment occurred from 2006 to 2010 . To focus on the acute phase of stroke, only the 2-week data are considered in the analyses described here.

\section{Participants}

All patients who had suffered an acute stroke and who were hospitalized at the study site were approached regarding participation. Inclusion criteria were i) ischemic stroke confirmed by brain magnetic resonance imaging (MRI) (or computed tomography (CT), if MRI was contraindicated); ii) ability to complete the necessary investigations and questionnaires; and iii) capacity to understand the objective of the study and provide informed consent. Exclusion criteria were i) severe physical illnesses that were life threatening or interfered with recovery from the stroke; ii) communication difficulties due to dysphasia or dysarthria, which precluded informed consent and questionnaire completion; iii) any of the following comorbid neuropsychiatric conditions: dementia, Parkinson's disease, brain tumor, epilepsy, psychoses, or alcohol or substance dependence; iv) severe physical illnesses limiting movement prior to stroke; and v) Korean Mini-Mental State Examination (K-MMSE) scores of $<16 .{ }^{24}$ All participants provided written informed consent, and the study was approved by the Institutional Review Board of CNUH.

\section{WHOQOL-BREF}

QOL was measured by the World Health Organization Quality of Life-Abbreviated form (WHOQOL-BREF), a 26-item self-administered questionnaire in which items are rated on a 5-point scale. WHOQOL-BREF evaluates domains related to physical factors, psychological factors, social relationships, and environmental context. Raw subscale scores are converted to a scale from 0 to 100 scale to facilitate comparisons with other data sets; higher scores indicate better QOL. The WHOQOLBREF was formerly standardized in Korean. ${ }^{25}$

\section{Socio-demographic data}

Data on age, sex, year of education, marital status, religious observation, and current occupation were obtained from participants or caregivers, as appropriate. Analyses employed the following categories: marital status (currently married vs. currently unmarried), religion (observant vs. non-observant), and occupation (employed vs. unemployed).

\section{Clinical characteristics}

Stroke severity was measured using the National Institutes of Health Stroke Scale (NIHSS). ${ }^{26}$ Scores on this instrument range from 0 and 42, and higher scores indicate more severe pathology. Physical disability was measured with the Barthel Index (BI). ${ }^{27}$ Scores on this instrument range from 0 to 100 , and lower scores indicate more severe disability. Cognitive functioning was evaluated by the K-MMSE, with scores ranging from 0 to 30, and lower scores indicating lower levels of cognitive functioning. The test was administered by a trained interviewer. Grip strength was measured in kilograms in both hands using a handheld dynamometer (T.K.K. 5401, Grip D; Takei Scientific Instruments Co, Japan). Measures for both hands were averaged to yield a composite measure of grip strength. Psychological distress was evaluated with the Hospital Anxiety and Depression Scale (HADS), which was divided into two subscales: the 7-item anxiety subscale (HADS-A) and the 7-item depression subscale (HADS-D). Each item is scored 
on 4-point scale (0-3), resulting in a total score of $0-21$ on each subscale, with higher scores indicating more severe pathology. ${ }^{28}$ The HADS was formerly standardized in Korean. ${ }^{29}$

\section{Statistical analyses}

Descriptive data are presented as means (standard deviations; range) or as numbers (percentages), as appropriate. The dependent variable was each WHOQOL-BREF domain continuous score. Initially, uni-variate associations were investigated between each QOL domain score and all independent socio-demographic and clinical characteristics using linear regression analyses. Factors significantly associated with each WHOQOL-BREF domain score on the uni-variate analyses ( $\mathrm{p}$-value $<0.05)$ were simultaneously entered into multi-variate analyses models to assess independence using multiple linear regression. The B-values and confidence intervals are presented as statistical coefficients. Statistical analyses were performed with SPSS 17.0 software (Statistical Package for the Social Sciences, SPSS, Inc., USA).

\section{RESULTS}

\section{Recruitment and descriptive data}

A total of 422 stroke patients who met the inclusion criteria were recruited. The mean (SD) time between stroke and assessment was 12.6 (3.3) days. Descriptive data on socio-demographic and clinical characteristics are presented in the first column of Table 1 . The mean (SD; range) scores of the four WHOQOL-BREF domains were as follows: physical factors, 47.5 (15.9; 3.6-85.7); psychological factors, 50.8 (16.6; 0-87.5); social relationships, 54.2 (11.8; 8.3-91.7); and environmental context, 46.1 (13.7; 0-81.3).

\section{Uni-variate associations of QOL with socio-demographic characteristics}

Associations between the four WHOQOL-BREF domain scores and socio-demographic characteristics are summarized in Table 2. Younger age, male sex, higher education, and current employment were significantly associated with higher scores on the physical factors. Male sex, higher education, and current

Table 1. Socio-demographic and clinical characteristics

\begin{tabular}{lc}
\hline Characteristics & Distribution in sample (N=422) \\
\hline Socio-demographic characteristics & $64.5(10.0 ; 30-87)$ \\
Age, mean (SD; range) years & $178: 244(57.8)$ \\
Sex, women : men (\%) & $8.6(4.9 ; 0-24)$ \\
Education, mean (SD; range) years & $103: 319(75.7)$ \\
Marital status, non-married : married (\%) & $189: 233(55.2)$ \\
Religion, no : yes (\%) & $229: 193(45.7)$ \\
Occupation, no : yes (\%) & $3.5(3.3 ; 0-18)$ \\
Clinical characteristics & $79.8(23.2 ; 0-100)$ \\
National Institutes of Health Stroke Scale, mean (SD; range) scores & $24.4(4.6 ; 11-30)$ \\
Barthel Index, mean (SD; range) scores & $22.2(11.3 ; 0-52)$ \\
Mini-mental state examination, mean (SD; range) scores & $4.6(4.2 ; 0-18)$ \\
Grip strength, mean (SD; range) & $2.3(3.1 ; 0-16)$ \\
Hospital Anxiety and Depression Scale-depression subscale, mean (SD; range) scores & \\
Hospital Anxiety and Depression Scale-anxiety subscale, mean (SD; range) scores &
\end{tabular}

Table 2. Uni-variate associations of quality of life with socio-demographic characteristics. Data are Bs (95\% confidence intervals)

\begin{tabular}{lcccc}
\hline & Physical factors & Psychological factors & Social relationships & Environmental context \\
\hline Age, higher & $-0.25(-0.40,-0.10)^{*}$ & $-0.16(-0.32,0.00)$ & $-0.07(-0.19,0.04)$ & $-0.08(-0.21,0.05)$ \\
Sex, women : men & $5.71(2.66,8.75)^{\ddagger}$ & $5.99(2.81,9.17)^{\ddagger}$ & $-1.57(-0.71,3.86)$ & $7.67(5.13,10.22)^{\ddagger}$ \\
Education, higher & $0.53(0.23,0.84)^{\dagger}$ & $0.81(0.501 .13)^{\ddagger}$ & $0.44(0.21,0.67)^{\ddagger}$ & $1.02(0.77,1.27)^{\ddagger}$ \\
Marital status, non-married : married & $3.21(-0.36,6.78)$ & $3.31(-0.41,7.02)$ & $0.93(-1.72,3.58)$ & $3.89(0.85,6.93)^{*}$ \\
Religion, no : yes & $-0.46(-3.53,2.60)$ & $-0.62(-3.82,2.59)$ & $0.75(-1.53,3.03)$ & $0.34(-2.30,2.98)$ \\
Occupation, no : yes & $5.21(2.15,8.26)^{\dagger}$ & $4.26(1.07,7.45)^{\dagger}$ & $0.94(-1.36,3.24)$ & $1.94(-0.69,4.58)$ \\
\hline
\end{tabular}

${ }^{*}$ p-value $<0.05,{ }^{\dagger} \mathrm{p}$-value $<0.01,{ }^{\ddagger} \mathrm{p}$-value $<0.001$ 
employment were significantly associated with higher scores on the psychological factors. Higher education was significantly associated with higher scores for social relationships. Male sex, higher education, and being married were significantly associated with higher scores on the environmental context.

\section{Uni-variate associations of QOL with clinical characteristics}

Associations between the four WHOQOL-BREF domain scores and clinical characteristics are summarized in Table 3. Lower NIHSS scores, higher BI and MMSE scores, stronger grip strength, and lower HADS-D and HADS-A scores were significantly associated with higher scores on the physical factors. Lower NIHSS scores, higher BI and MMSE scores, stronger grip strength, and lower HADS-D and HADS-A scores were significantly associated with higher scores on the psychological factors. Higher BI scores, stronger grip strength, and lower HADS-D and HADS-A scores were significantly associated with higher scores for social relationships. Lower NIHSS scores, higher BI and MMSE scores, stronger grip strength, and lower HADS-D and HADS-A scores were significantly associ- ated with higher scores on the environmental context.

\section{Multivariate associations with QOL}

Adjusted associations between the four WHOQOL-BREF domain scores and simultaneously entered independent variables are summarized in Table 4. Higher MMSE scores, stronger grip strength, and lower HADS-D and HADS-A scores were independently associated with higher scores on the physical factors. More education, higher MMSE scores, and lower HADS-D and HADS-A scores were independently associated with higher scores on the psychological factors. Lower HADS-D and HADS-A scores were independently associated with higher scores for social relationships. More education, higher MMSE scores, and lower HADS-D scores were independently associated with higher scores on the environmental context.

\section{DISCUSSION}

The principal findings of the present study of inpatients experiencing the acute period following stroke were as fol-

Table 3. Uni-variate associations of quality of life with clinical characteristics. Data are Bs (95\% confidence intervals)

\begin{tabular}{lcccc}
\hline & Physical factors & Psychological factors & Social relationships & Environmental context \\
\hline NIHSS, higher & $-1.19(-1.65,-0.74)^{\ddagger}$ & $-0.65(-1.14,-0.17)^{\dagger}$ & $-0.35(-0.70,0.00)$ & $-0.45(-0.86,-0.05)^{*}$ \\
BI, higher & $0.20(0.13,0.26)^{\ddagger}$ & $0.13(0.06,0.20)^{\ddagger}$ & $0.07(0.02,0.12)^{\dagger}$ & $0.07(0.01,0.13)^{*}$ \\
MMSE, higher & $0.60(0.27,0.93)^{\ddagger}$ & $0.69(0.35,1.04)^{\ddagger}$ & $0.21(-0.04,0.46)$ & $0.64(0.36,0.93)^{\ddagger}$ \\
Grip strength, higher & $0.59(0.43,0.69)^{\ddagger}$ & $0.46(0.32,0.60)^{\ddagger}$ & $0.21(0.11,0.31)^{\ddagger}$ & $0.40(0.29,0.52)^{\ddagger}$ \\
HADS-D, higher & $-2.32(-2.61,-2.04)^{\ddagger}$ & $-2.46(-2.76,-2.17)^{\ddagger}$ & $-1.05(-1.30,-0.80)^{\ddagger}$ & $-1.65(-1.91,-1.38)^{\ddagger}$ \\
HADS-A, higher & $-2.27(-2.71,-1.81)^{\ddagger}$ & $-2.67(-2.74,-1.79)^{\ddagger}$ & $-1.19(-1.54,-0.84)^{\ddagger}$ & $-1.26(-1.67,-0.85)^{\ddagger}$ \\
\hline
\end{tabular}

${ }^{*}$ p-value $<0.05,{ }^{\dagger}$-value $<0.01,{ }^{\ddagger}$-value $<0.001$. NIHSS: National Institutes of Health Stroke Scale, BI: Barthel Index, MMSE: Mini-Mental State Examination, HADS-D: Hospital Anxiety and Depression Scale depression subscale, HADS-A: Hospital Anxiety and Depression Scale anxiety subscale

Table 4. Multi-variate associations of quality of life. Data are Bs (95\% confidence intervals)

\begin{tabular}{lcccc}
\hline & Physical factors & Psychological factors & Social relationships & Environmental context \\
\hline Age, higher & $0.01(-0.13,0.16)$ & - & - & - \\
Sex, women : men & $-0.91(-4.24,2.42)$ & $-0.45(-3.89,2.98)$ & - & $1.76(-1.34,4.85)$ \\
Education, higher & $0.04(-0.27,0.34)$ & $0.39(0.07,0.71)^{\dagger}$ & $0.22(-0.03,0.47)$ & $0.77(0.48,1.06)^{\ddagger}$ \\
Marital status, non-married : married & - & - & - & $0.07(-2.81,2.95)$ \\
Occupation, no : yes & $0.88(-1.76,3.51)$ & $-0.55(-3.29,2.18)$ & - & - \\
NIHSS, higher & $-0.30(-0.89,0.29)$ & $0.11(-0.75,0.53)$ & - & $-0.14(-0.71,0.44)$ \\
BI, higher & $0.06(-0.02,0.14)$ & $0.03(-0.07,0.12)$ & $0.03(-0.02,0.08)$ & $0.01(-0.07,0.09)$ \\
MMSE, higher & $0.44(0.12,0.76)^{\dagger}$ & $0.42(0.08,0.76)^{*}$ & - & $0.33(0.03,0.63)^{*}$ \\
Grip strength, higher & $0.33(0.17,0.49)^{\ddagger}$ & $0.15(-0.01,0.31)$ & $0.03(-0.09,0.14)$ & $0.08(-0.07,0.22)$ \\
HADS-D, higher & $-1.74(-2.11,-1.37)^{\ddagger}$ & $-2.15(-2.55,-1.74)^{\ddagger}$ & $-0.67(-1.01,-0.34)^{\ddagger}$ & $-1.41(-1.76,-1.06)^{\ddagger}$ \\
HADS-A, higher & $-0.79(-1.25,-0.32)^{\ddagger}$ & $-0.52(-1.03,-0.02)^{*}$ & $-0.63(-1.06,-0.20)^{\dagger}$ & $-0.09(-0.54,0.36)$ \\
\hline
\end{tabular}

${ }^{*}$ p-value $<0.05,{ }^{\dagger}$-value $<0.01,{ }^{\ddagger}$-value $<0.001$. NIHSS: National Institutes of Health Stroke Scale, BI: Barthel Index, MMSE: Mini-Mental State Examination, HADS-D: Hospital Anxiety and Depression Scale depression subscale, HADS-A: Hospital Anxiety and Depression Scale anxiety subscale 
lows: 1) psychological distress, as evaluated by the HADS, was consistently associated with all four QOL domains measured by the WHOQOL-BREF; 2) cognitive functioning (MMSE scores) was independently associated with three QOL domains; 3) NIHSS and BI scores were not associated with any QOL domains; and 4) education was the only socio-demographic variable that was independently associated with the psychological and environmental domains of the WHOQOLBREF.

It is noteworthy that previous studies on QOL following stroke have focused primarily on long-term outcomes rather than on the acute phase. As mentioned above, many psychological, demographic, and physical factors contribute to longterm QOL after acute stroke. However, few studies on the factors that predict QOL in stroke patients during the acute phase have been conducted. Kwok et al. ${ }^{16}$ studied the determinants of QOL in Chinese stroke survivors during the 3-12 months after discharge and found that environment and social interaction were associated with QOL. Additionally, Jonsson et al. ${ }^{17}$ underscored the role of depressive symptoms and functional status in determining QOL relatively shortly after discharge for stroke. The Extremity Constraint-Induced Therapy Evaluation (EXCITE) trial found that demographic factors such as age, sex, education, comorbid conditions, stroke type, and dominant hand were predictors of the QOL of patients with mild to moderate strokes during the subacute stage (3-9 months). ${ }^{18}$ Although these studies investigated the determinants of QOL in acute stroke patients within relatively short-term follow-up periods compared with the periods examined by other studies, they were not able to assess the factors associated with QOL during the acute phase (especially during admission).

This limitation may be attributable to the fact that clinicians attend to saving lives and treating the neurological problems of patients who have suffered from an acute stroke rather than to QOL. In general, neurological management is given priority in the treatment of stroke patients during the acute phase, and psychological and rehabilitative considerations become more important during the rehabilitation or chronic phases of stroke. However, we focused on associations between QOL and other factors during the acute phase of stroke. Patients in the current study were stroke survivors who were assessed within 2 weeks of the stroke. Additional studies with larger samples can provide more information about the determinants of QOL in the acute stage of stroke.

We expected that neurological symptoms such as stroke severity, impaired ability to perform ADLs, and cognitive functioning would be the primary factors associated with QOL in patients who have survived strokes, but we eventually found that psychological distress such as depression and anxiety was most strongly and consistently associated with all QOL domains, even during the acute phase. Depression, which has been identified in $30 \%$ of patients following stroke, has been correlated with QOL in this population. ${ }^{30,31}$ It has been suggested that depression usually develops 6-12 months after stroke and can continue for up to 3 years following this event. ${ }^{32,33}$ Several long-term studies have examined the role of depression and anxiety as a determinants of QOL after stroke. . $^{17,19,20}$ The findings of these long-term studies are consistent with those of the present study (higher HADS-A and -D scores among stroke patients).

Cognitive functioning, as evaluated by the MMSE, was also independently associated with the physical, psychological, and environmental domains of the WHOQOL-BREF. A few studies have reported that cognitive functioning was independently associated with QOL in stroke survivors during a long-term follow-up period. ${ }^{19,22,34}$ These findings suggest that a psychiatric perspective, in addition to neurological management, is important during the acute phase of stroke. However, given that we interviewed all enrolled patients during admission and found a higher prevalence of post-stroke depression 6 months after discharge, reevaluations are needed after the post-discharge period.

Physical disability has consistently been identified as a determinant of QOL among stroke survivors in almost all studies. Unexpectedly, NIHSS and BI scores, the main indices of stroke severity, were not associated with any domains of the WHOQOL-BREF in the present study. These findings may result from our focus on the acute stage of stroke in that nearly all patients were bed ridden, and associations of measures of stroke severity, such as the NIHSS and BI, with QOL cannot be assessed as precisely during the acute stage as during the chronic stage of stroke. However, NIHSS was known to be a well-reflecting marker of severity of acute stage rather than chronic stage of stroke. Because severity of acute stroke can be underestimated due to anosognosia in patients with right middle cranial arterial territory infarction, further evaluation is needed to seek association QOL with stroke severity of acute stroke. Alternatively, this finding can be interpreted as suggesting the importance of psychological versus physical factors during the acute stage of stroke. Of the variables used in assessing neurological symptoms, only grip strength was independently associated with the physical domain of QOL. Grip strength has been known to be associated with the QOL of elderly persons, ${ }^{35}$ and weaker grip strength has been correlated with decreased QOL among those who have suffered from an ischemic stroke. ${ }^{36}$ Consequently, grip strength, rather than other markers of physical disability such as NIHSS and $\mathrm{BI}$ scores, can be used as a predictor of QOL during the acute stage of stroke. In our previous report from the same dataset, depression and anxiety was not associated with stroke severity assessed by NIHSS and BI. ${ }^{37}$ These results suggested that psychological distress may influence QOL directly rather than via stroke 
severity indirectly.

Education was associated with the psychological and environmental domains of the WHOQOL-BREF in the current study. The NEMESIS study and the EXCITE trial reported that less education was associated with decreased QOL, and this finding suggests that educational status is an independent predictor of QOL during both the acute and chronic stages after an acute stroke. ${ }^{18,21}$ In our present study, there are more male patients in study populations. Several studies have found that women who survived strokes have less favorable outcomes than do their male counterparts. Women are less likely to be discharged to their homes than are men and are more likely to have physical impairments and limitations in their daily living. ${ }^{38}$ Additionally, women have lower overall QOL and lower levels of functional recovery compared with those of males. ${ }^{39,40}$ The Tinzaparin in Acute Ischemic Stroke Trial (TAIST) reported that being female was predictive of poor functional outcomes in stroke. ${ }^{41}$ Thus, separate examinations of male and female stroke survivors should be performed.

Our study has several strengths. First, QOL and the other important variables were evaluated using well standardized instruments at a similar time point (two weeks after stroke) in all participants. The present study focused on the acute post-stroke period, and therefore reduced the risk of bias arising from heterogeneous time since stroke. Second, participants were consecutive patients with a recent stroke from the study hospital, which reduced the likelihood of selection bias and increases the potential generalizability. However, an important consideration in interpreting the present findings is that participants with severe cognitive impairment or aphasia who could not perform the self rating scales or psychiatric interview were excluded, and therefore the findings can only be assumed to apply to people with mild to moderate stroke severity without these deficits. Another consideration, this analysis evaluated QOL in only acute stroke patents, and therefore long-term follow-up study is needed. ${ }^{42}$

In conclusion, the present study demonstrated that psychological distress and impaired cognitive functioning were associated with QOL in stroke survivors during the acute phase following stroke. However, stroke severity, physical disability and other socio-demographic factors did not significantly influence QOL in these individuals. These findings underscore the importance of psychological as well as neurological interventions during the acute phase following stroke.

\section{Acknowledgments}

This research was supported by Basic Science Research Program through the National Research Foundation of Korea (NRF) funded by the Ministry of Education, Science and Technology (2009-0087344).

\section{REFERENCES}

1. Roger VL, Go AS, Lloyd-Jones DM, Adams RJ, Berry JD, Brown TM, et al. Heart disease and stroke statistics--2011 update: a report from the American Heart Association. Circulation 2011;123:e18-e209.

2. Asplund K, Stegmayr B, Peltonen M. From the Twentieth to the Twenty-First Century: A Public Health Perspective on Stroke. In: Ginsberg MD, Bogousslavsky J, Editors. Cerebrovascular Disease: Pathophysiology, Diagnosis, and Management. Vol 2. Malden, MA: Blackwell Science; 1998.

3. The WHOQOL Group. Development of the World Health Organization WHOQOL-BREF quality of life assessment. Psychol Med 1998; 28:551-558.

4. Schipper H, Clinch JJ, Olweny CLM. Quality of Life Studies: Definitions and Conceptual Issues. In: Spilker B, Editor. Quality of Life and Pharmacoeconomics in Clinical Trials. New York: Lippincott-Raven, 1996, p. 11-23.

5. World Health Organization. WHO STEPS Stroke Manual: The WHO STEPwise Approach to Stroke Surveillance. Geneva: WHO; 2006.

6. MacKenzie AE, Chang AM. Predictors of quality of life following stroke. Disabil Rehabil 2002;24:259-265.

7. Burton CR. Living with stroke: a phenomenological study. J Adv Nurs 2000;32:301-309.

8. Kim P, Warren S, Madill H, Hadley M. Quality of life of stroke survivors. Qual Life Res 1999;8:293-301.

9. Niemi ML, Laaksonen R, Kotila M, Waltimo O. Quality of life 4 years after stroke. Stroke 1988;19:1101-1107.

10. de Haan RJ, Limburg M, Van der Meulen JH, Jacobs HM, Aaronson NK. Quality of life after stroke. Impact of stroke type and lesion location. Stroke 1995;26:402-408.

11. Carod-Artal J, Egido JA, Gonzalez JL, Varela de Seijas E. Quality of life among stroke survivors evaluated 1 year after a stroke: experience of a stroke unit. Stroke 2000;31:2995-3000.

12. Williams LS, Kroenke K, Weinberger M, Harris LE, Biller J. Post-stroke depression affects quality of life (QOL) but not other stroke outcome measures. Stroke 1999;30:236-239.

13. Astrom M, Asplund K, Astrom T. Psychosocial function and life satisfaction after stroke. Stroke 1992;23:527-531.

14. King RB. Quality of life after stroke. Stroke 1996;27:1467-1472.

15. Carod-Artal FJ, Egido JA. Quality of life after stroke: the importance of a good recovery. Cerebrovasc Dis 2009;27(Suppl 1):204-214.

16. Kwok T, Lo RS, Wong E, Wai-Kwong T, Mok V, Kai-Sing W. Quality of life of stroke survivors: a 1-year follow-up study. Arch Phys Med Rehabil 2006;87:1177-1182.

17. Jonsson AC, Lindgren I, Hallstrom B, Norrving B, Lindgren A. Determinants of quality of life in stroke survivors and their informal caregivers. Stroke 2005;36:803-808.

18. Nichols-Larsen DS, Clark PC, Zeringue A, Greenspan A, Blanton S. Factors influencing stroke survivors' quality of life during subacute recovery. Stroke 2005;36:1480-1484.

19. Haacke C, Althaus A, Spottke A, Siebert U, Back T, Dodel R. Longterm outcome after stroke: evaluating health-related quality of life using utility measurements. Stroke 2006;37:193-198.

20. Naess H, Waje-Andreassen U, Thomassen L, Nyland H, Myhr KM. Health-related quality of life among young adults with ischemic stroke on long-term follow-up. Stroke 2006;37:1232-1236.

21. Paul SL, Sturm JW, Dewey HM, Donnan GA, Macdonell RA, Thrift AG. Long-term outcome in the North East Melbourne Stroke Incidence Study: predictors of quality of life at 5 years after stroke. Stroke 2005;36: 2082-2086.

22. Patel MD, McKevitt C, Lawrence E, Rudd AG, Wolfe CD. Clinical determinants of long-term quality of life after stroke. Age Ageing 2007; 36:316-322.

23. Kim JM, Stewart R, Bae KY, Kim SW, Kang HJ, Shin IS, et al. Serotonergic and BDNF genes and risk of depression after stroke. J Affect 
Disord 2012;136:833-840.

24. Kang YW, Na DL, Han SH. A validity study on the Korean Mini-Mental State Examination (K-MMSE) in dementia patients. J Korean Neurol Assoc 1997;15:300-308.

25. Min SK, Lee CI, Kim KI, Suh SY, Kim DK. Development of Korean version of WHO Quality of Life Scale Abbreviated Version (WHOQOL-BREF). J Korean Neuropsychiatr Assoc 2000;39:571-579.

26. Kasner SE, Chalela JA, Luciano JM, Cucchiara BL, Raps EC, McGarvey ML, et al. Reliability and validity of estimating the NIH stroke scale score from medical records. Stroke 1999;30:1534-1537.

27. Mahoney FI, Barthel DW. Functional evaluation: the Barthel Index. Md State Med J 1965;14:61-65.

28. Zigmond AD, Snaith RP. The hospital anxiety and depression scale. Acta Psychiatr Scand 1983;67:361-370.

29. Oh SM, Min KJ, Park DB. A study on the standardization of the Hospital Anxiety and Depression Scale for Koreans: a comparison of normal, depressed and anxious groups. J Korean Neuropsychiatr Assoc 1999;38:289-296.

30. Bluvol A, Ford-Gilboe M. Hope, health work and quality of life in families of stroke survivors. J Adv Nurs 2004;48:322-332.

31. Carod-Artal FJ. Post-stroke depression (I). Epidemiology, diagnostic criteria and risk factors. Rev Neurol 2006;42:169-175.

32. Bogousslavsky J. William Feinberg lecture 2002: emotions, mood, and behavior after stroke. Stroke 2003;34:1046-1050.

33. Whyte EM, Mulsant BH. Post stroke depression: epidemiology, pathophysiology, and biological treatment. Biol Psychiatry 2002;52:253-264.

34. Kwa VI, Limburg M, de Haan RJ. The role of cognitive impairment in the quality of life after ischemic stroke. J Neurol 1996;243:599-604.

35. Sayer AA, Syddall HE, Martin HJ, Dennison EM, Roberts HC, Cooper C. Is grip strength associated with health-related quality of life? Find- ings from the Hertfordshire Cohort Study. Age Ageing 2006;35:409415.

36. Muller-Nordhorn J, Nolte CH, Rossnagel K, Jungehulsing GJ, Reich A, Roll S, et al. The use of the 12-item short-term health status instrument in a longitudinal study of patients with stroke and transient ischaemic attack. Neuroepidemiology 2005;24:196-202.

37. Kang HJ, Bae KY, Kim SW, Kim JM, Shin IS, Kim JT, et al. Prevalence and risk factors of post-stroke depression. J Korean Soc Depress Bipolar Disord 2011:9:57-63.

38. Grenthe Olsson B, Sunnerhagen KS. Functional and cognitive capacity and health-related quality of life 2 years after day hospital rehabilitation for stroke: a prospective study. J Stroke Cerebrovasc Dis 2007;16: 208-215.

39. Carod-Artal FJ, Gonzalez-Gutierrez JL, Herrero JA, Horan T, De Seijas EV. Functional recovery and instrumental activities of daily living: follow-up 1-year after treatment in a stroke unit. Brain Inj 2002;16:207216.

40. Gargano JW, Reeves MJ; Paul Coverdell National Acute Stroke Registry Michigan Prototypes Investigators. Sex differences in stroke recovery and stroke-specific quality of life: results form a statewide stroke registry. Stroke 2007;38:2541-2548.

41. Gray LJ, Sprigg N, Bath PM, Boysen G, De Deyn PP, Leys D, et al. Sex differences in quality of life in stroke survivors: data from the Tinzaparin in Acute Ischaemic Stroke Trial (TAIST). Stroke 2007;38:29602964.

42. Kim JM, Stewart R, Kim SW, Yang SJ, Shin IS, Kim YH, et al. Interactions between life stressors and susceptibility genes (5-HTTLPR and BDNF) on depression in Korean elders. Biol Psychiatry 2007;62:423428. 